\title{
Robotic-assisted non-intubated tracheal resection: the most excellent care or an unnecessary surgical challenge?
}

\author{
Paolo A. Ferrari ${ }^{1}$, Alessandro Tamburrini ${ }^{2}$ \\ ${ }^{1}$ Division of Thoracic Surgery, “A. Businco” Oncology Hospital, A.R.N.A.S. “G. Brotzu”, Cagliari, Italy; ${ }^{2}$ Division of Thoracic Surgery, University \\ Hospital Southampton NHS Foundation Trust, Southampton, UK \\ Correspondence to: Paolo A. Ferrari. Division of Thoracic Surgery, “A. Businco” Cancer Center, A.R.N.A.S. “G. Brotzu”, Via Jenner 1, 09121, Cagliari, \\ Italy. Email: paoloalb.ferrari@gmail.com. \\ Comment on: Li S, Ai Q, Liang H, et al. Non-intubated Robotic-Assisted Thoracic Surgery for Tracheal/Airway Resection and Reconstruction: \\ Technique Description and Preliminary Results. Ann Surg 2021. [Epub ahead of print]. doi: 10.1097/SLA.0000000000004887.
}

Submitted Sep 10, 2021. Accepted for publication Sep 30, 2021.

doi: $10.21037 /$ atm-21-4686

View this article at: https://dx.doi.org/10.21037/atm-21-4686

Minimally invasive resection and reconstruction of the airways performed in non-intubated patients are technically challenging and significantly demanding surgical procedures, which certainly require a considerable set of skills and high expertise.

History has however constantly taught us that limits only exist to be overcome, and the remarkable advances achieved by thoracic surgeons in recent decades represent for us a continuous reminder.

In 2018, the "Guangzhou Institute of Respiratory Health" team first reported on the feasibility of thoracoscopic resection and reconstruction of trachea and airways in spontaneously ventilating patients, with benefits in terms of improved anastomosis and shorter operative time $(1,2)$.

Following these encouraging results, the same team introduced a novel technique of tracheal and tracheabronchial resection and reconstruction by robotic-assisted thoracic surgery (RATS) in non-intubated patients. The authors must be applauded and deserve outstanding merit for their preliminary report, but in order to truly appreciate the innovation of Shuben and colleagues (3), it is helpful to take a step back.

Single-lung ventilation allowing surgeons to operate on a collapsed lung has been considered so far mandatory for most thoracic surgery operations. Nevertheless, the recent increasing demand for less invasive approaches alongside improved safety and effectiveness of surgical procedures, resulted in a process of constant aim for enhanced technology and ameliorated techniques by the thoracic surgeons and the anesthesiologists of our era. In this scenario, non-intubated minimally-invasive video-assisted thoracic surgery appeared to raise and stand as the perfect combination of these concepts (4).

It is furthermore well established that the implementation of minimally-invasive techniques has proven clear benefits for patients undergoing thoracic procedures, making videoassisted-thoracic surgery (VATS) the standard of care for a wide variety of operations (5), including complex airway surgery (6).

In addition, the prominent role of anesthesia in thoracic surgery must be taken into account, as it equally contributes alongside surgical techniques and operative skills, to the accomplishment of a "successful" result (7).

The avoidance of the side-effects of traditional general anesthesia and invasive ventilation, provides indeed a more physiologic cardiopulmonary, muscular, and neurological status (8). Supported by this rationale, in the past two decades some thoracic surgeons started to shift the paradigm from standard single-lung ventilation to thoracic procedures performed in selected, non-intubated and spontaneously ventilating patients. Katlic described a remarkable series of over 500 patients operated under local anesthesia and sedations only, entailing lung biopsies, pleural operations and other minor procedures (9). Other centers performed non-intubated thymectomy and lung resections (10), "awake" video-assisted thoracoscopic resection of lung nodules with multiport or single- 
port approaches $(11,12)$, and uniportal non-intubated lobectomies (13), utilizing different analgesia strategies including local blocks or epidural catheters, and avoidance of intravenous neuromuscular blocking agents, usually in combination with supraglottic devices for airways support (laryngeal masks).

In order to thoroughly understand the criticisms and the advantages of non-intubated thoracic surgery, it is of paramount importance to consider the airway management and the anesthetic process from the anesthesiologist's perspective. An optimal level of analgesia and adequate level of consciousness/sedation must be carefully maintained and balanced throughout the operation, as patients should be responsive enough to provide a reasonable response to verbal or tactile stimulation, and not only pain reflexes. Additionally, no interventions to maintain adequate spontaneous ventilation or cardiovascular function should be required during the surgery. The whole process requires a remarkable level of expertise and strong collaboration between the surgeon and the anesthesiologist, certainly more compared to traditional general anesthesia and invasive ventilation for major thoracic surgery (14).

In this article, Shuben and colleagues (3) meticulously selected patients requiring resection for tracheobronchial neoplasms, and carefully managed them with intravenous propofol+remifentanil+sufentanil, targeting the bispectral index to allow spontaneous ventilation. Muscle relaxants were avoided, while a laryngeal mask was used to maintain the airways. An experienced anesthesiologist continuously monitored the patients and was prepared and ready to intubate in the lateral position, should the need arise. The postoperative outcomes showed favorable results, satisfactory anastomotic healing, no major complications and no stenosis nor stricture of the anastomosis.

In these patients, the reported advantages of this airways management technique in addition to the minimallyinvasive approach are still a topic of debate (15), and can be essentially summarized in quicker recovery and decreased physiological stress (16).

Additionally, the technical benefits of the non-intubated setting in airway surgery provides a more effortless management of the actual airway anastomosis, as there is no interference from the endotracheal tube in the surgical field (1). This translates also into a valuable time-saving step in the RATS procedures, as the repositioning of the robotic arms during cross-field ventilation is no longer required.
Conversely, the bulky presence of robotic arms may represent a reason for criticism, if in an emergency scenario rapid tracheal intubation should be required. As always, clear communication between the surgical and the anesthetic teams is essential to ensure patient's safety, successful surgery, and optimal postoperative pain control.

Nevertheless, non-intubated airway surgery is still infrequent, and the benefits over traditional intubated surgery remain unclear, especially for carinal and lower tracheal resections and reconstructions, procedures in which where mortality rates remain high (15).

On the other hand, the approach to tracheobronchial surgery is also rapidly evolving through technological advances. Combined with tubeless anesthesia, RATS may promote the utilization of a minimally-invasive approach for more complex and technically demanding thoracic operations, succeeding where VATS has been rather slow instead to achieve a broader use (17).

We certainly look forward to more studies comparing rates of utilizations, outcomes, and benefits between RATS and VATS for both intubated and non-intubated settings in patients undergoing tracheal and trachea-bronchial resections.

As more surgeons gain familiarity with the minimallyinvasive approaches to airway surgery and more anesthesiologists improve their non-intubated management skills, we anticipate that more patients will benefit from these fascinating and innovative techniques.

\section{Acknowledgments}

Funding: None.

\section{Footnote}

Provenance and Peer Review: This article was commissioned by the editorial office, Annals of Translational Medicine. The article did not undergo external peer review.

Conflicts of Interest: Both authors have completed the ICMJE uniform disclosure form (available at https://dx.doi. org/10.21037/atm-21-4686). The authors have no conflicts of interest to declare.

Ethical Statement: The authors are accountable for all aspects of the work in ensuring that questions related to the accuracy or integrity of any part of the work are 
appropriately investigated and resolved.

Open Access Statement: This is an Open Access article distributed in accordance with the Creative Commons Attribution-NonCommercial-NoDerivs 4.0 International License (CC BY-NC-ND 4.0), which permits the noncommercial replication and distribution of the article with the strict proviso that no changes or edits are made and the original work is properly cited (including links to both the formal publication through the relevant DOI and the license). See: https://creativecommons.org/licenses/by-nc-nd/4.0/.

\section{References}

1. Jiang L, Liu J, Gonzalez-Rivas D, et al. Thoracoscopic surgery for tracheal and carinal resection and reconstruction under spontaneous ventilation. J Thorac Cardiovasc Surg 2018;155:2746-54.

2. Liang H, Gonzalez-Rivas D, Zhou Y, et al. Nonintubated Anesthesia for Tracheal/Carinal Resection and Reconstruction. Thorac Surg Clin 2020;30:83-90.

3. Li S, Ai Q, Liang H, et al. Non-intubated Robotic-Assisted Thoracic Surgery for Tracheal/Airway Resection and Reconstruction: Technique Description and Preliminary Results. Ann Surg 2021. [Epub ahead of print]. doi: 10.1097/SLA.0000000000004887.

4. Rocco G. Non-intubated uniportal lung surgery†. Eur J Cardiothorac Surg 2016;49 Suppl 1:i3-5.

5. Oparka JD, Yan TD, Walker WS. Twenty years of videoassisted thoracoscopic surgery: The past, present, and future. Thorac Cancer 2013;4:91-4.

6. Li J, Wang W, Jiang L, et al. Video-Assisted Thoracic Surgery Resection and Reconstruction of Carina and Trachea for Malignant or Benign Disease in 12 Patients: Three Centers' Experience in China. Ann Thorac Surg 2016;102:295-303

Cite this article as: Ferrari PA, Tamburrini A. Roboticassisted non-intubated tracheal resection: the most excellent care or an unnecessary surgical challenge? Ann Transl Med 2021;9(21):1632. doi: 10.21037/atm-21-4686
7. Tamburrini A, Mineo TC. A glimpse of history: non-intubated thoracic surgery. Video-assist Thorac Surg 2017;2:52.

8. Pompeo E. State of the art and perspectives in nonintubated thoracic surgery. Ann Transl Med 2014;2:106.

9. Katlic MR. Five Hundred Seventy-Six Cases of VideoAssisted Thoracic Surgery Using Local Anesthesia and Sedation: Lessons Learned. J Am Coll Surg 2018; 226:58-63.

10. Al-Abdullatief M, Wahood A, Al-Shirawi N, et al. Awake anaesthesia for major thoracic surgical procedures: an observational study. Eur J Cardiothorac Surg 2007; 32:346-50.

11. Pompeo E, Mineo D, Rogliani P, et al. Feasibility and results of awake thoracoscopic resection of solitary pulmonary nodules. Ann Thorac Surg 2004;78:1761-8.

12. Rocco G, Romano V, Accardo R, et al. Awake singleaccess (uniportal) video-assisted thoracoscopic surgery for peripheral pulmonary nodules in a complete ambulatory setting. Ann Thorac Surg 2010;89:1625-7.

13. Gonzalez-Rivas D, Aymerich H, Bonome C, et al. From Open Operations to Nonintubated Uniportal VideoAssisted Thoracoscopic Lobectomy: Minimizing the Trauma to the Patient. Ann Thorac Surg 2015; 100:2003-5.

14. Liu J, Cui F, Li S, et al. Nonintubated video-assisted thoracoscopic surgery under epidural anesthesia compared with conventional anesthetic option: a randomized control study. Surg Innov 2015;22:123-30.

15. Okuda K, Nakanishi R. The non-intubated anesthesia for airway surgery. J Thorac Dis 2016;8:3414-9.

16. Mineo TC, Tacconi F. From "awake" to "monitored anesthesia care" thoracic surgery: A 15 year evolution. Thorac Cancer 2014;5:1-13.

17. Cohen BD, Marshall MB. Robotic-assisted tracheobronchial surgery. J Thorac Dis 2020;12:6173-8. 